\title{
Subclinical acute kidney injury is associated with adverse outcomes in critically ill neonates and children
}

Fang Fang ${ }^{1 \dagger}$, Xiaohan $\mathrm{Hu}^{1 \dagger}$, Xiaomei Dai ${ }^{2}$, Sanfeng Wang ${ }^{2}$, Zhenjiang $\mathrm{Bai}^{3}$, Jiao Chen ${ }^{3}$, Jian Pan ${ }^{1}$, Xiaozhong $\mathrm{Li}^{2}$, Jian Wang ${ }^{1}$ and Yanhong $\mathrm{Li}^{1,2^{*}}$ (D)

\begin{abstract}
Background: Research on acute kidney injury (AKI) has focused on identifying early biomarkers. However, whether AKI could be diagnosed in the absence of the classic signs of clinical AKI and whether the condition of subclinical AKl, identified by damage or functional biomarkers in the absence of oliguria or increased serum creatinine $(\mathrm{s} C \mathrm{r})$ levels, is clinically significant remains to be elucidated in critically ill children. The aims of the study were to investigate the associations between urinary cystatin $\mathrm{C}(\mathrm{UCys} \mathrm{C}$ ) levels and AKI and mortality and to determine whether uCysCpositive subclinical AKI is associated with adverse outcomes in critically ill neonates and children.

Methods: In this prospective cohort study, uCysC levels were serially measured during the first week after intensive care unit (ICU) admission in a heterogeneous group of patients $(n=510)$ presenting to a tertiary neonatal and pediatric ICU. The diagnosis of neonatal AKI that developed during the first week after admission was based on neonatal KDIGO criteria or $\mathrm{s} C r>1.5 \mathrm{mg} / \mathrm{dL}$, and pediatric AKI was based on Kidney Disease: Improving Global Outcomes (KDIGO) criteria. The term "uCysC(-)" or "uCysC(+)", indicating the absence or presence of tubular injury, was defined by the optimal peak uCysC cutoff value for predicting ICU mortality.

Results: The initial and peak uCysC levels were significantly associated with AKI and mortality, and had an area under the receiver operating characteristic curve of 0.76 and 0.81 , respectively, for predicting mortality. At the optimal cutoff value of $1260 \mathrm{ng} / \mathrm{mg} \mathrm{uCr}$, the peak uCysC displayed sensitivity of $79.2 \%$ and specificity of $72.3 \%$ for predicting mortality. Among all patients, 130 (25.5\%) developed uCysC(+)/AKI(-) status during the first week after admission. The adjusted odds ratio for patients with uCys $C(+) / A K I(-)$ status in association with an increased risk of mortality compared with that for patients with uCysC(-)/AKI(-) was $9.34(P<0.001)$. Patients with uCysC(+)/AKI(-) spent 2.8 times as long in the ICU as those with uCysC $(-) / \mathrm{AKI}(-)(P<0.001)$.

Conclusions: Both initial and peak UCysC levels are associated with $\mathrm{AKI}$ and mortality and are independently predictive of mortality in critically ill neonates and children. Subclinical AKI may occur without detectable loss of kidney function, and UCysC-positive subclinical AKI is associated with worse clinical outcomes in this population.
\end{abstract}

Keywords: Acute kidney injury, Adverse outcomes, Critically ill neonates, Critically ill children, Length of ICU stay, Mortality, Subclinical acute kidney injury, Urinary cystatin C

\footnotetext{
* Correspondence: lyh072006@hotmail.com

${ }^{\dagger}$ Fang Fang and Xiaohan Hu contributed equally to this work.

${ }^{1}$ Institute of Pediatric Research, Children's Hospital of Soochow University,

Suzhou, JiangSu province, China

${ }^{2}$ Department of Nephrology, Children's Hospital of Soochow University,

Suzhou, JiangSu province, China

Full list of author information is available at the end of the article
}

(c) The Author(s). 2018 Open Access This article is distributed under the terms of the Creative Commons Attribution 4.0 International License (http://creativecommons.org/licenses/by/4.0/), which permits unrestricted use, distribution, and

reproduction in any medium, provided you give appropriate credit to the original author(s) and the source, provide a link to the Creative Commons license, and indicate if changes were made. The Creative Commons Public Domain Dedication waiver (http://creativecommons.org/publicdomain/zero/1.0/) applies to the data made available in this article, unless otherwise stated. 


\section{Background}

Acute kidney injury (AKI) is associated with adverse outcomes in critically ill children, such as longer hospital stay and increased mortality, [1-3]. Research on AKI has focused on identifying early biomarkers capable of detecting kidney injury before the rise in serum creatinine $(\mathrm{sCr})$ being observed in recent decades, which could optimize and improve patient outcomes [4-8]. Previous studies have demonstrated that the diagnosis of clinical AKI is delayed by at least 24-48 hours compared to diagnosis of the condition identified by using novel renal biomarkers of tubular injury, such as neutrophil gelatinase-associated lipocalin (NGAL) $[9,10]$ and cystatin C (CysC) $[10,11]$. Therefore, questions arise regarding whether AKI could be diagnosed in the absence of the classic signs of clinical AKI and whether the condition of subclinical AKI, identified by damage or functional biomarkers in the absence of oliguria or increased $\mathrm{sCr}$ levels, is clinically significant. Recent studies suggest that subclinical AKI, which precedes clinical AKI and is defined as tubular injury in the absence of oliguria or increased $\mathrm{sCr}$ levels, is still AKI [12-15] and has an increased risk of adverse outcomes in adult patients [15]. However, whether subclinical AKI may occur frequently in critically ill neonates and children and whether the development of subclinical AKI in this population is associated with negative clinical outcomes remains to be elucidated.

Cystatin C, a $13-\mathrm{kDa}$ protein, is normally filtered freely and is completely reabsorbed and catabolized in the proximal tubule and therefore is not normally found in urine in significant amounts. Increased levels of CysC in urine may reflect renal tubular injury and impairment [16, 17]. Urinary CysC ( $\mathrm{uCysC}$ ) has been shown to be diagnostic of AKI in patients in the intensive care unit (ICU) $[11,18$, 19]. Our previous studies demonstrated that $\mathrm{uCysC}$ is associated with AKI and is independently predictive of AKI in critically ill neonates $[20,21]$. Increases in $\mathrm{uCysC}$ levels predict AKI 24-48 hours before the occurrence of diagnostic $\mathrm{sCr}$ increases in response to renal injury $[10,11]$, suggesting that $\mathrm{uCysC}$ may identify subclinical kidney injury, in which kidney damage occurs without the development of decreased kidney function. However, whether uCysC could provide a method of detecting subclinical AKI in patients with apparently normal $\mathrm{sCr}$ is unknown. It also remains unclear whether $\mathrm{uCys} C$ is associated with increased mortality in critically ill neonates and children with and without AKI. The aims of the study were to investigate the association between uCysC level and AKI and mortality in critically ill neonates and children, to evaluate whether uCysC could detect subclinical AKI in patients with apparently normal sCr, and to determine whether uCysC-positive subclinical AKI was associated with adverse outcomes in this population.

\section{Methods}

\section{Subject selection}

All patients admitted to the neonatal and pediatric intensive care unit (NICU and PICU, respectively) from July to October 2016 were eligible for this prospective study. A priori exclusion criteria were known congenital abnormality of the kidney and failure to collect urine samples before discharge from the ICU or death. This study was approved by the Institutional Review Board at the Children's Hospital of Soochow University and performed in accordance with the Declaration of Helsinki. Informed parental consent was obtained at enrollment.

\section{Clinical data collection}

We reviewed the medical records of eligible patients. Clinical status, medication, and therapies were recorded daily until ICU discharge or death. Sepsis, multiple organ dysfunction syndrome (MODS), shock, and disseminated intravascular coagulation (DIC) that developed during the ICU stay were diagnosed by the treating physicians, according to the criteria described previously [22, 23].

\section{Assessment of illness severity}

The score for neonatal acute physiology (SNAP) and the score of the pediatric risk of mortality III (PRISM III), which were calculated on the day of NICU or PICU admission according to methods described in the original study [24, 25] and in our previous studies [22, 26], were used as a measure to assess illness severity.

\section{Diagnosis of AKI}

The diagnosis of neonatal AKI that developed during the first week after admission was based on (1) the neonatal AKI Kidney Disease: Improving Global Outcome (KDIGO) classification, i.e., $\mathrm{sCr}$ rise $\geq 0.3 \mathrm{mg} / \mathrm{dL}(26.5$ $\mu \mathrm{mol} / \mathrm{L}$ ) within $48 \mathrm{~h}, \mathrm{sCr}$ rise $\geq 1.5$ times the reference $\mathrm{sCr}$ within 7 days, or urine output $\leq 0.5 \mathrm{~mL} / \mathrm{kg} / \mathrm{h}$ for $6-12 \mathrm{~h}$ (the reference $\mathrm{sCr}$ was defined as the lowest previous $\mathrm{sCr}$ value) [27] or (2) $\mathrm{sCr}>1.5 \mathrm{mg} / \mathrm{dL}(132.6 \mu \mathrm{mol} / \mathrm{L})$ sustained at least $48 \mathrm{~h}$ [20]. The KDIGO criteria for $\mathrm{sCr}$ level and urine output were applied to define pediatric AKI that developed during the first week after PICU admission [28]. Baseline sCr was defined as the lowest level obtained within 3 months prior to ICU admission [1]. When the baseline $\mathrm{sCr}$ measurement was unavailable, the $\mathrm{sCr}$ value at the time of enrollment was used. For patients with elevated $\mathrm{sCr} \geq 1.2 \mathrm{mg} / \mathrm{dL}(106.1 \mu \mathrm{mol} / \mathrm{L})$ at PICU admission, the lowest $\mathrm{sCr}$ value within 2 weeks while in the PICU was considered the baseline $\mathrm{sCr}$, which was adapted from a previous study [29] and was in accordance with our previous study [22]. When the two criteria resulted in different KDIGO stages, the higher stage was chosen. Severity of AKI was characterized by KDIGO staging. Severe AKI was defined as KDIGO stage 2 or 3 [1]. 


\section{Outcomes}

The primary outcome was ICU mortality defined as all-cause mortality occurring during the ICU stay, including death resulting from withdrawal of therapy. Secondary outcomes were the duration of mechanical ventilation (MV) and the ICU length of stay.

\section{Measurement of urinary CysC}

Urine samples were collected every 48-72 hours during the first week after the first collection on the first day of being admitted to the ICU and immediately frozen and stored at $-80^{\circ} \mathrm{C}$. For the measurement of $\mathrm{CysC}$, the urine sample was centrifuged at $1500 \mathrm{~g}$ at $4{ }^{\circ} \mathrm{C}$ for $10 \mathrm{~min}$, and the supernatants were aliquoted and measured using an automatic biochemical analyzer (Hitachi 7600, Tokyo, Japan) and a latex enhanced immunoturbidimetry assay, as previously described $[20,21]$. The detection limit for CysC was $10 \mathrm{ng} / \mathrm{mL}$. The intra-assay and inter-assay coefficients of variation were $\leq 10 \%$, corresponding to that reported by the manufacturer. Urinary CysC value was expressed in nanograms per milligram of urinary $\mathrm{Cr}(\mathrm{ng} / \mathrm{mg} \mathrm{uCr})$. The $\mathrm{uCr}$ level from the same aliquoted sample was measured automatically using the sarcosine oxidase method.

\section{Statistical analysis}

Statistical analyses were performed using SPSS statistics. We first checked the assumptions of normality and homogeneity of variance. Skewed distributions are described by the median and interquartile range (IQR) and were compared using the Mann-Whitney U test or the Kruskal-Wallis $\mathrm{H}(\mathrm{K})$ test. Categorical variables are presented as counts and percentages, and Fisher's exact test or the chi-square test was applied for comparison, as appropriate. Stepwise multivariate linear regression analyses were performed to investigate factors potentially associated with the levels of uCysC. Univariate and multivariate logistic regression analyses were performed to calculate the odds ratio (OR) and adjusted OR (AOR) with a 95\% confidence interval (CI) to assess the association between variables and AKI and mortality. To analyze the predictive power, the receiver operating characteristic (ROC) curves were generated, the area under the ROC curve (AUC) was determined, and the nonparametric method of Delong was used to compare differences between AUCs. The sensitivity, specificity, positive likelihood ratio (LR+), and negative likelihood ratio (LR-) were calculated using Sigma Plot 10.0 software. Univariate and multivariate linear regression analyses were performed to investigate the association between $\mathrm{uCysC}$ and the duration of $\mathrm{MV}$ and the length of ICU stay. Continuous variables with skewed distributions were log-transformed for linear regression analysis. The two-sided alpha level was set at 0.05 .

\section{Results}

\section{Patient characteristics}

The prospective study involved 510 patients, including 239 neonates from the NICU and 271 children between 1 month and 16 years of age from the PICU. Of the total of 517 patients admitted to the NICU and PICU during the study period, 7 were excluded because of a failure in collecting urine samples before discharge from the ICU or death. The leading cause of ICU admission in the cohort was respiratory disease $(36.5 \%)$, followed by neurologic disease (11.8\%). Fifty-six patients (11.0\%) were clinically diagnosed with sepsis.

Of the 510 patients, 79 (15.5\%) developed AKI during the first week after admission, including 43 (8.4\%) who fulfilled the criteria for KDIGO stage 1, which is defined as mild AKI, and 36 (7.1\%) patients who fulfilled the criteria for KDIGO stages 2 and 3, which are defined as severe AKI. The ICU mortality rate in the whole cohort with or without AKI was 48 (9.4\%; 95\% CI 6.9-12.0\%) during the ICU stay. None of the patients had any known congenital abnormality of the kidney. One patient received aminoglycosides (oral streptomycin) during the ICU stay. A comparison of the demographic and clinical characteristics between survivors and non-survivors is displayed in Table 1.

\section{Urinary CysC}

Of the 510 patients, 120 (23.5\%) had one sample, 139 (27.3\%) had two samples, and 251 (49.2\%) had three samples available during the first week after ICU admission. The missing samples were because of patients being discharged from the ICU or death and failure to collect urine. The urinary $\mathrm{CysC}$ (uCysC) levels were detectable in 1083 samples (94.1\%). Samples with undetectable uCysC were assigned a uCysC value of $5 \mathrm{ng} /$ $\mathrm{mL}$, which is equivalent to half of the detection limit of the assay, to facilitate the calculation of $\mathrm{uCysC} / \mathrm{uCr}$ ratios. The initial and the peak values of uCysC were used for analysis of association. For each patient, the level of CysC from the urinary sample collected on the first day of admission to ICU was denoted as the initial (first) uCysC. The highest uCysC level among all collected samples was denoted the peak uCysC.

\section{Correlation between urinary $\mathrm{CysC}$ level and clinical variables}

There was significant correlation between the initial and peak levels of $\mathrm{uCysC}$ and age, body weight, illness severity, duration of MV, AKI stage, sepsis, MODS, and the use of vancomycin, assessed using Spearman's correlation analysis, as shown in Table 2. Furthermore, variables with a $P$ value $<0.1$ (shown in Table 2 ) were entered in stepwise multiple linear regression analysis to investigate factors potentially associated with the uCysC levels. As shown in 
Table 1 Comparison of demographic and clinical characteristics between survivors and non-survivors

\begin{tabular}{|c|c|c|c|}
\hline & Survivors, $n=462$ & Non-survivors, $n=48$ & $P$ value \\
\hline Age, months & $1.37[0.33-16.0]$ & $0.97[0.33-13.5]$ & 0.540 \\
\hline \multicolumn{4}{|l|}{ Age group, $n$} \\
\hline$\leq 28$ days, $n=239$ & $215(46.5)$ & $24(50.0)$ & \multirow[t]{3}{*}{0.893} \\
\hline$\leq 1$ year, $n=132$ & $120(26.0)$ & $12(25.0)$ & \\
\hline$>1$ year, $n=139$ & $127(27.5)$ & $12(25.0)$ & \\
\hline Body weight, kg & $4.2[2.5-11.0]$ & $2.9[1.5-10.0]$ & 0.035 \\
\hline Male, $n$ & $277(60.0)$ & $27(56.3)$ & 0.645 \\
\hline Illness severity ${ }^{a}$, score & 6 [3-9] & $10[6-14]$ & $<0.001$ \\
\hline \multicolumn{4}{|l|}{ Diagnosis on ICU admission } \\
\hline Respiratory diseases, $n$ & $176(38.1)$ & $10(20.8)$ & \multirow[t]{8}{*}{0.054} \\
\hline Neurologic diseases, $n$ & $59(12.8)$ & $1(2.1)$ & \\
\hline Preterm/LBW, $n$ & $37(8.0)$ & $5(10.4)$ & \\
\hline Sepsis, $n$ & $45(9.7)$ & $11(22.9)$ & \\
\hline Poison/trauma/accident, $n$ & $34(7.4)$ & $5(10.4)$ & \\
\hline Cardiologic diseases, $n$ & $20(4.3)$ & $1(2.1)$ & \\
\hline Gastroenterologic, $n$ & $19(4.1)$ & $0(0)$ & \\
\hline Hematologic diseases, $n$ & $17(3.7)$ & $6(12.5)$ & \\
\hline $\mathrm{MV}^{\mathrm{b}}, n$ & $127(27.5)$ & $35(72.9)$ & $<0.001$ \\
\hline MV duration, days & $0[0-0.84]$ & $2.23[0-6.86]$ & $<0.001$ \\
\hline MV duration $\geq 48$ hours, $n$ & $89(19.3)$ & $24(50.0)$ & $<0.001$ \\
\hline$A K I^{c}, n$ & 63 (13.6) & $16(33.3)$ & 0.002 \\
\hline AKI stage $1, n$ & $36(7.8)$ & $7(14.6)$ & \multirow[t]{3}{*}{$<0.001$} \\
\hline AKI stage $2, n$ & $19(4.1)$ & $5(10.4)$ & \\
\hline AKI stage $3, n$ & $8(1.7)$ & $4(8.3)$ & \\
\hline Sepsis $^{\mathrm{b}}, n$ & $58(9.7)$ & $15(22.9)$ & 0.001 \\
\hline Shock/DIC,$n$ & $61(13.2)$ & $13(27.1)$ & 0.013 \\
\hline MODS $^{b}, n$ & $65(14.1)$ & $14(29.2)$ & 0.009 \\
\hline Furosemide $^{b}, n$ & $126(27.3)$ & $24(50.0)$ & 0.002 \\
\hline Steroid $^{b}, n$ & $150(32.5)$ & $21(43.8)$ & 0.147 \\
\hline Antibiotics $^{\mathrm{b}}, n$ & $418(90.5)$ & $46(95.8)$ & 0.294 \\
\hline Vancomycin ${ }^{\mathrm{b}}, n$ & $35(7.6)$ & $7(14.6)$ & 0.099 \\
\hline Mannitol $^{b}, n$ & $115(24.9)$ & $16(33.3)$ & 0.225 \\
\hline Inotrope $^{b}, n$ & $60(13.0)$ & $10(20.8)$ & 0.182 \\
\hline Hemofiltration $^{\mathrm{b}}, n$ & $15(3.2)$ & $3(6.3)$ & 0.235 \\
\hline Initial uCysC, ng/mg uCr & 311.82 [122.22-975.34] & 1587.39 [583.19-8749.16] & $<0.001$ \\
\hline Peak uCysC, ng/mg uCr & 426.49 [165.23-1607.62] & 7026.73 [1285.25-33879.56] & $<0.001$ \\
\hline
\end{tabular}

Values are median [interquartile range] or number (percentage)

AKI acute kidney injury, DIC disseminated intravascular coagulation, ICU intensive care unit, LBW low birth weight, MODS multi-organ dysfunction syndrome, MV mechanical ventilation, $u C y s C$ urinary cystatin $C, u C r$ urinary creatinine

allness severity was assessed by the score for neonatal acute physiology in critically ill neonates and the pediatric risk of mortality III score in critically ill children

${ }^{\mathrm{b}}$ Administered or developed during ICU stay

'Developed during the first week after ICU admission

Table 3, illness severity, body weight, AKI stage, MODS, and age were identified as independent factors significantly associated with the initial uCysC levels (total $R^{2}=0.25$ ).
The levels of peak uCysC were independently associated with illness severity, body weight, MODS, AKI stage, age, and duration of MV (total $R^{2}=0.35$ ). 
Table 2 Spearman's analysis of correlation between urinary cystatin C and clinical variables $(n=510)$

\begin{tabular}{|c|c|c|c|c|}
\hline & \multicolumn{2}{|c|}{ Initial urinary cystatin C } & \multicolumn{2}{|c|}{ Peak urinary cystatin C } \\
\hline & Spearman's $r$ & $P$ value & Spearman's $r$ & $P$ value \\
\hline Age, months & -0.230 & $<0.001$ & -0.307 & $<0.001$ \\
\hline Body weight, kg & -0.302 & $<0.001$ & -0.389 & $<0.001$ \\
\hline Male, $n$ & -0.005 & 0.904 & -0.024 & 0.589 \\
\hline Illness severity ${ }^{a}$, score & 0.358 & $<0.001$ & 0.415 & $<0.001$ \\
\hline $\mathrm{MV}^{\mathrm{b}}, n$ & 0.087 & 0.051 & 0.128 & 0.004 \\
\hline Duration of MV, days & 0.093 & 0.035 & 0.141 & 0.001 \\
\hline$A K I^{c}, n$ & 0.172 & $<0.001$ & 0.164 & $<0.001$ \\
\hline AKI stage & 0.182 & $<0.001$ & 0.175 & $<0.001$ \\
\hline Severe $A K I^{d}, n$ & 0.197 & $<0.001$ & 0.196 & $<0.001$ \\
\hline Sepsis $^{\mathrm{b}}, n$ & 0.040 & 0.362 & 0.065 & 0.141 \\
\hline Shock/DIC,$n$ & 0.060 & 0.173 & 0.076 & 0.086 \\
\hline MODS $^{b}, n$ & 0.102 & 0.021 & 0.122 & 0.006 \\
\hline Furosemide ${ }^{b}, n$ & 0.070 & 0.112 & 0.127 & 0.004 \\
\hline Steroid $^{\mathrm{b}}, n$ & -0.020 & 0.655 & -0.005 & 0.919 \\
\hline Antibiotics ${ }^{\mathrm{b}}, n$ & 0.080 & 0.072 & 0.106 & 0.017 \\
\hline Vancomycin $^{\mathrm{b}}, \mathrm{n}$ & 0.106 & 0.016 & 0.121 & 0.006 \\
\hline Mannitol $^{b}, n$ & -0.041 & 0.350 & -0.070 & 0.112 \\
\hline Inotrope $^{b}, n$ & 0.023 & 0.606 & 0.034 & 0.444 \\
\hline Hemofiltration $^{\mathrm{b}}, n$ & 0.007 & 0.880 & -0.006 & 0.898 \\
\hline
\end{tabular}

$r=$ Spearman's correlation coefficient

$A K I$ acute kidney injury, DIC disseminated intravascular coagulation, ICU intensive care unit, MODS multi-organ dysfunction syndrome, $M V$ mechanical ventilation allness severity was assessed by the score for neonatal acute physiology in critically ill neonates and the pediatric risk of mortality III score in critically ill children

${ }^{\mathrm{b}}$ Administered or developed during ICU stay

cDeveloped during the first week after ICU admission

${ }^{\mathrm{d}}$ Severe AKI was defined as Kidney Disease: Improving Global Outcomes (KDIGO) stages 2 and 3

Table 3 Clinical variables potentially associated with urinary cystatin C levels $(n=510)^{*}$

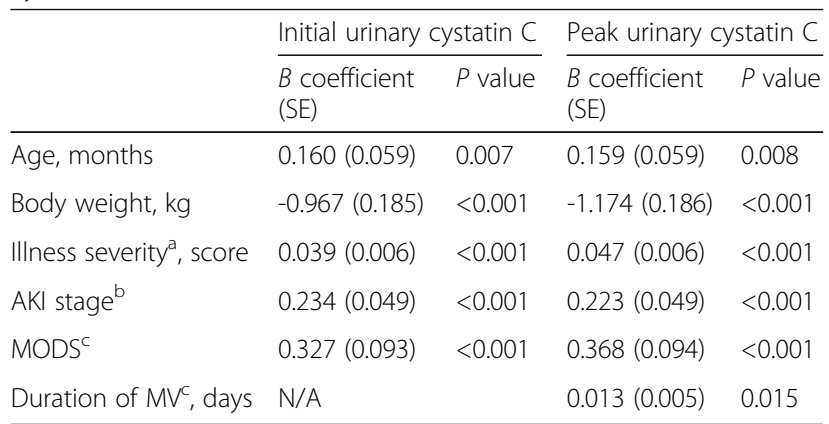

*Variables with a $P$ value $<0.1$ (shown in Table 2 ) were entered into the stepwise multivariate linear regression analysis. Continuous variables were log-transformed

MODS multi-organ dysfunction syndrome, MV mechanical ventilation, N/A not applicable

allness severity was assessed by the score for neonatal acute physiology in critically ill neonates and the pediatric risk of mortality III score in critically ill children

beveloped during the first week after ICU admission

${ }^{\mathrm{c}}$ Administered or developed during ICU stay

\section{Association between urinary CysC and AKI}

To identify whether uCysC was independently associated with AKI that developed during the first week after ICU admission in critically ill neonates and children, the variables in Table 1 were analyzed by logistic regression. As shown in Table 4, both the initial and peak values of uCysC were significantly associated with AKI. The association between initial (adjusted OR $(\mathrm{AOR})=1.12$ per $10,000 \mathrm{ng} / \mathrm{mg} \mathrm{uCr}$ increase, $95 \% \mathrm{CI} 1.02-1.23, P=0.024)$ and peak $(\mathrm{AOR}=1.11$ per $10,000 \mathrm{ng} / \mathrm{mg} \mathrm{uCr}$ increase, 95\% CI 1.02-1.21, $P=0.014)$ uCysC with AKI remained significant after adjustment for age, body weight, illness severity, and MV. A comparison of the initial and the peak values of uCysC among patients grouped according to AKI stage is displayed in Additional file 1: Table S1.

The performance of $\mathrm{uCysC}$ in predicting AKI and severe AKI is displayed in Table 5 . The initial and peak values of $\mathrm{uCysC}$ were predictive of severe AKI with an AUC of 0.72 for predicting severe AKI. The ROC curves of the initial and peak uCysC for predicting severe AKI are displayed in Fig. 1. Peak uCysC had sensitivity of $61.1 \%$ and specificity of $76.0 \%$ at the optimal cutoff value of $1736 \mathrm{ng} / \mathrm{mg} \mathrm{uCr}$ for predicting severe AKI in critically ill neonates and children, as shown in Table 5.

\section{Association between urinary CysC and ICU mortality}

To identify whether uCysC was independently associated with increased risk of death in critically ill neonates and children, variables with a $P$ value $<0.05$ (shown in the comparison in Table 1), which were considered confounding factors, were analyzed by univariate and multivariate logistic regression. Univariate analysis identified that illness severity, the use and duration of MV, AKI diagnosis and stage, sepsis, shock/DIC, MODS, the use of furosemide, and the initial and peak values of $\mathrm{uCysC}$ were significantly associated with mortality. The odds ratio for predicting morality is shown in Table 6. The association between the initial $(\mathrm{AOR}=1.13$ per $10,000 \mathrm{ng} / \mathrm{mg} \mathrm{uCr}$ increase, 95\% CI 1.01-1.26, $P=0.041$ ) and the peak $(\mathrm{AOR}=1.17$ per $10,000 \mathrm{ng} / \mathrm{mg} \mathrm{uCr}$ increase, $95 \% \mathrm{CI}$ 1.07-1.28, $P<0.001$ ) values of uCysC and mortality remained significant after adjustment for potential confounders including body weight, illness severity, MV, and AKI stage in multivariate logistic analysis, as displayed in Table 6. A comparison of the mortality rate by quintile of the peak uCysC level is shown in Additional file 2: Figure $\mathrm{S} 1 \mathrm{~A}$. The association between $\mathrm{uCysC}$ and clinical variables and ICU mortality in critically ill neonates and children, respectively, is shown in Additional file 1: Table S2.

The performance of $\mathrm{uCysC}$ in predicting mortality is assessed in Table 7. Both initial and peak values of uCysC predicted mortality. Peak uCysC $(\mathrm{AUC}=0.81, \mathrm{P}$ 
Table 4 Association between urinary cystatin C or clinical variables and AKI that developed during the first week after ICU admission $(n=510)$

\begin{tabular}{|c|c|c|c|c|c|c|}
\hline & OR & $95 \% \mathrm{Cl}$ & $P$ value & $\mathrm{AOR}$ & $95 \% \mathrm{Cl}$ & $P$ value \\
\hline Age, months & 1.01 & $1.00-1.01$ & 0.001 & $1.00^{d}$ & $0.98-1.01$ & 0.592 \\
\hline Body weight, kg & 1.04 & $1.02-1.06$ & $<0.001$ & $1.06^{\mathrm{e}}$ & $0.99-1.13$ & 0.123 \\
\hline Illness severity ${ }^{a}$, score & 1.12 & $1.07-1.16$ & $<0.001$ & $1.11^{\mathrm{f}}$ & $1.06-1.16$ & $<0.001$ \\
\hline$M V^{b}$ & 3.13 & $1.92-5.12$ & $<0.001$ & $2.02^{9}$ & $1.16-3.51$ & 0.013 \\
\hline MV duration, days & 1.05 & $1.02-1.08$ & 0.002 & $1.02^{h}$ & $0.98-1.06$ & 0.292 \\
\hline Shock/DIC ${ }^{b}$ & 2.81 & $1.59-4.98$ & $<0.001$ & $1.84^{h}$ & $0.98-3.44$ & 0.058 \\
\hline MODS $^{b}$ & 3.50 & $2.02-6.07$ & $<0.001$ & $1.87^{\mathrm{h}}$ & $0.99-3.51$ & 0.053 \\
\hline Furosemide $^{b}$ & 3.85 & $2.35-6.32$ & $<0.001$ & $2.84^{h}$ & $1.62-4.98$ & $<0.001$ \\
\hline Steroid $^{\mathrm{b}}$ & 1.84 & $1.13-2.99$ & 0.015 & $1.07^{\mathrm{h}}$ & $0.60-1.91$ & 0.821 \\
\hline Initial uCysC, ng/mg uCr & $1.18^{\mathrm{c}}$ & $1.07-1.31$ & 0.001 & $1.12^{\mathrm{c}, \mathrm{h}}$ & $1.02-1.23$ & 0.024 \\
\hline Peak uCysC, ng/mg uCr & $1.18^{c}$ & $1.09-1.28$ & $<0.001$ & $1.11^{\mathrm{c}, \mathrm{h}}$ & $1.02-1.21$ & 0.014 \\
\hline
\end{tabular}

$A K I$ acute kidney injury, $A O R$ adjusted $O R, C I$ confidence interval, DIC disseminated intravascular coagulation, $I C U$ intensive care unit, $M O D S$ multi-organ

dysfunction syndrome, $M V$ mechanical ventilation, $O R$ odds ratio, $u C y s C$ urinary cystatin $C, u C r$ urinary creatinine

allness severity was assessed by the score for neonatal acute physiology in critically ill neonates and the pediatric risk of mortality III score in critically ill children

${ }^{\mathrm{b}}$ Administered or developed during ICU stay

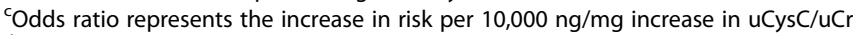

${ }^{\mathrm{d}}$ After adjustment for body weight and illness severity

${ }^{\mathrm{e}}$ After adjustment for age and illness severity

${ }^{f}$ After adjustment for age and body weight

${ }^{g}$ After adjustment for age, body weight, and illness severity

${ }^{h}$ After adjustment for age, body weight, illness severity, and mechanical ventilation

$<0.001)$ was better than initial uCysC (AUC $=0.76, P<$ 0.001 ) or illness severity score (AUC $=0.74, P<0.001$ ) in predicting mortality. However, the difference was not statistically significant. The $P$ value for the comparison of the AUCs between peak uCysC and illness severity score and between peak and initial uCysC was 0.161 and 0.273 , respectively. The $\mathrm{ROC}$ curves of $\mathrm{uCysC}$ and illness severity score for predicting the mortality of patients are displayed in Fig. 2. We also calculated the optimal cutoff values of $\mathrm{uCysC}$ to predict mortality. Peak $\mathrm{uCysC}$ had sensitivity of $79.2 \%$ and specificity of $72.3 \%$ at the optimal cutoff value of $1260 \mathrm{ng} / \mathrm{mg} \mathrm{uCr}$ in critically ill neonates and children (Table 7). The predictive characteristics of $\mathrm{uCysC}$ at different cutoff values for ICU mortality in critically ill neonates and children are displayed in Additional file 1: Table S3. The performance of $\mathrm{uCysC}$ in predicting ICU mortality in critically ill neonates and children, respectively, is displayed in Additional file 1: Table S4.

\section{Association between urinary $\mathrm{Cys} C$ and secondary outcomes}

Both the initial and the peak values of $\mathrm{uCys} C$ were associated with the duration of MV (initial, unstandardized coefficient $B=0.202$, SE $=0.069, P=0.003$; peak, $B=0.277$, $\mathrm{SE}=0.064, P<0.001)$ and the length of the ICU stay (initial, $B=0.159, \mathrm{SE}=0.028, P<0.001$; peak, $B=0.231$, $\mathrm{SE}=0.025, P<0.001$ ) by using univariate linear regression analysis. The peak $\mathrm{uCysC}$, but not the initial uCysC, remained significantly associated with the duration of $\mathrm{MV}$ ( $B=0.183$, SE $=0.071, P=0.010)$ and the length of the ICU stay $(B=0.110, \mathrm{SE}=0.025, P<0.001)$ after adjustment for body weight and illness severity in multivariate linear regression analysis. Univariate and multivariate linear regression analyses were performed with log-transformed data on the duration of MV and length of the ICU stay. Patients who did not receive MV were arbitrarily given a value of -2 . A comparison of the length of the ICU stay by quintile of

Table 5 Predictive characteristics of urinary cystatin C for AKI or severe AKI $(n=510)$

\begin{tabular}{|c|c|c|c|c|c|c|c|c|}
\hline uCysC, ng/mg uCr & AUC & $95 \% \mathrm{Cl}$ & $P$ value & Optimal cutoff value & Sensitivity & Specificity & LR+ & LR- \\
\hline \multicolumn{9}{|l|}{ AKI } \\
\hline Initial uCysC & 0.64 & $0.57-0.71$ & $<0.001$ & 1788.0 & $38.0 \%$ & $85.2 \%$ & 2.6 & 0.73 \\
\hline Peak uCysC & 0.63 & $0.56-0.70$ & $<0.001$ & 8816.0 & $30.4 \%$ & $93.3 \%$ & 4.5 & 0.75 \\
\hline \multicolumn{9}{|l|}{ Severe AKI } \\
\hline Initial uCysC & 0.72 & $0.63-0.82$ & $<0.001$ & 3389.0 & $50.0 \%$ & $89.1 \%$ & 4.6 & 0.56 \\
\hline Peak uCysC & 0.72 & $0.63-0.81$ & $<0.001$ & 1736.0 & $61.1 \%$ & $76.0 \%$ & 2.5 & 0.51 \\
\hline
\end{tabular}

$A K I$ acute kidney injury, $A U C$ the area under the ROC curve, $C l$ confidence interval, $L R+$ positive likelihood ratio, $L R$ - negative likelihood ratio, $u C y s C$ urinary cystatin $C$ AKI developed during the first week after ICU admission. Kidney Disease: Improving Global Outcomes (KDIGO) stage 2 or 3 was defined as severe AKI 


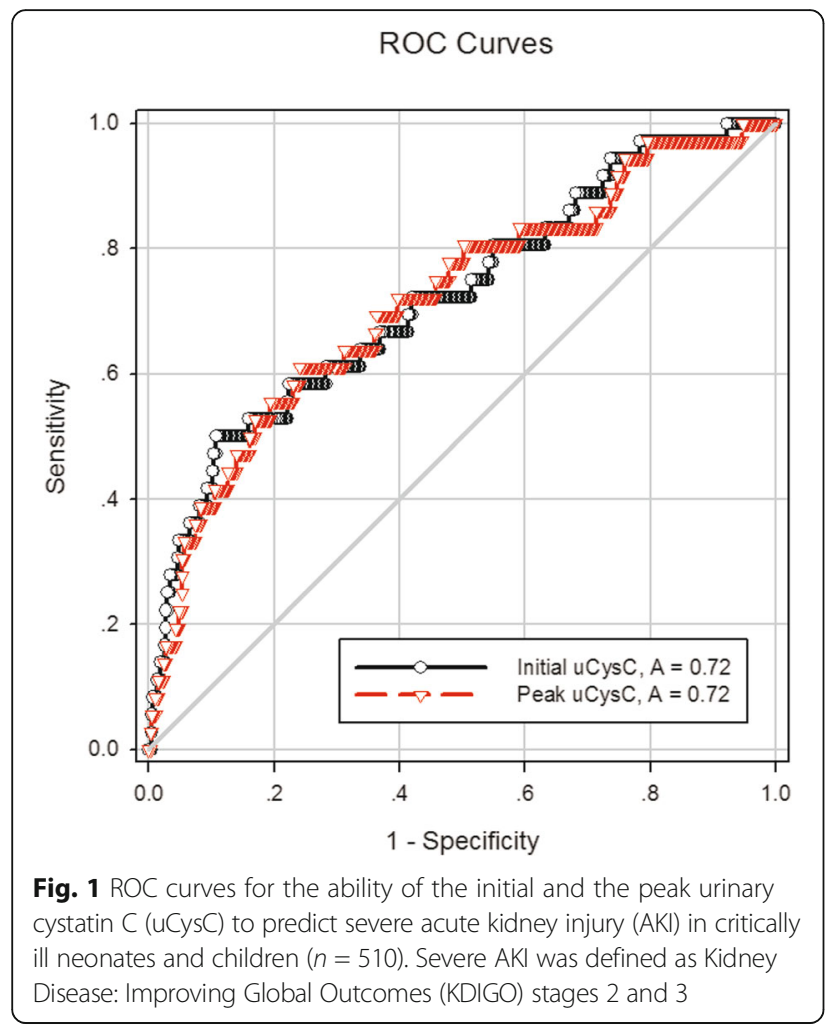

the peak $\mathrm{uCys} C$ level is shown in Additional file 2: Figure S1B.

\section{Association between urinary CysC-positive subclinical AKI and ICU mortality}

To identify whether uCysC-positive subclinical AKI that developed during the first week after admission was associated with increased risk of adverse outcomes in patients, "uCysC(-)" and "uCysC(+)", indicating the absence and presence of tubular injury, were defined by the optimal cutoff value of peak uCysC $(1260 \mathrm{ng} / \mathrm{mg}$ $\mathrm{uCr}$ ) for predicting mortality. Patients were classified as follows: uCysC(-)/AKI(-), uCysC(+)/AKI(-), uCysC(-)/ $\mathrm{AKI}(+)$, and $\mathrm{uCysC}(+) / \mathrm{AKI}(+)$. The clinical characteristics and outcomes grouped according to these four groups are shown in Table 8. Among 510 patients, 25.5\% developed $\mathrm{uCysC}(+) / \mathrm{AKI}(-)$ status, which was much more common than $\mathrm{uCysC}(-) / \mathrm{AKI}(+)$. Mortality in patients with $\mathrm{uCysC}(+) / \mathrm{AKI}(-)$ status was significantly higher than that in patients with $\mathrm{uCysC}(-) / \mathrm{AKI}(-)$. The difference remained significant after adjusting for body weight and illness severity $(P<0.001)$. Although mortality in patients with $\mathrm{uCysC}(+) / \mathrm{AKI}(-)$ tended to be higher than that in patients with $\mathrm{uCysC}(-) / \mathrm{AKI}(+)$ status, the difference did not reach significant $(P=0.162)$. There was also no significant difference in mortality between patients with $\mathrm{uCysC}(+) / \mathrm{AKI}(-)$ and those with uCysC $(+) / \mathrm{AKI}(+)(P=0.116)$. Mortality was significantly

Table 6 Association between urinary cystatin C or clinical variables and ICU mortality $(n=510)$

\begin{tabular}{|c|c|c|c|c|c|c|}
\hline & OR & $95 \% \mathrm{Cl}$ & $P$ value & AOR & $95 \% \mathrm{Cl}$ & $P$ value \\
\hline Body weight, kg & 1.00 & $0.97-1.03$ & 0.968 & $0.99^{\mathrm{e}}$ & $0.95-1.02$ & 0.454 \\
\hline Illness severity ${ }^{a}$, score & 1.14 & $1.09-1.19$ & $<0.001$ & $1.14^{\mathrm{f}}$ & $1.09-1.20$ & $<0.001$ \\
\hline$M V^{b}$ & 7.10 & $3.64-13.86$ & $<0.001$ & $4.72^{\mathrm{g}}$ & $2.28-9.76$ & $<0.001$ \\
\hline MV duration, days & 1.09 & $1.05-1.12$ & $<0.001$ & $1.07^{9}$ & $1.04-1.11$ & $<0.001$ \\
\hline$A K l^{c}$ & 3.17 & $1.64-6.10$ & 0.001 & $2.09^{9}$ & $0.94--3.97$ & 0.049 \\
\hline AKI stage & 1.87 & $1.34-2.60$ & $<0.001$ & $1.49^{9}$ & $1.00-4.33$ & 0.031 \\
\hline Severe $\mathrm{AKI}^{\mathrm{C}}$ & 3.72 & $1.63-8.46$ & 0.002 & $2.25^{9}$ & $0.91-5.57$ & 0.079 \\
\hline Sepsis ${ }^{b}$ & 3.17 & $1.62-6.18$ & 0.001 & $2.58^{h}$ & $1.22-5.45$ & 0.013 \\
\hline Shock/DIC ${ }^{b}$ & 2.44 & $1.22-4.87$ & 0.011 & $1.32^{\mathrm{h}}$ & $0.59-2.96$ & 0.500 \\
\hline MODS $^{b}$ & 2.52 & $1.28-4.94$ & 0.007 & $1.18^{\mathrm{h}}$ & $0.51-2.72$ & 0.706 \\
\hline Furosemide $^{b}$ & 2.67 & $1.46-4.87$ & 0.001 & $1.09^{h}$ & $0.53-2.23$ & 0.814 \\
\hline Initial uCysC, ng/mg uCr & $1.19^{d}$ & $1.08-1.33$ & 0.001 & $1.13^{\mathrm{d}, \mathrm{h}}$ & $1.01-1.26$ & 0.041 \\
\hline Peak uCysC, ng/mg uCr & $1.26^{\mathrm{d}}$ & $1.16-1.38$ & $<0.001$ & $1.17^{\mathrm{d}, \mathrm{h}}$ & $1.07-1.28$ & $<0.001$ \\
\hline
\end{tabular}

AKI acute kidney injury, $A O R$ adjusted $O R, C l$ confidence interval, ICU intensive care unit, DIC disseminated intravascular coagulation, MODS, multi-organ dysfunction syndrome, $M V$ mechanical ventilation, $O R$ odds ratio, $u C y s C$ urinary cystatin $C, u C r$ urinary creatinine

allness severity was assessed by the score for neonatal acute physiology in critically ill neonates and the pediatric risk of mortality III score in critically ill children

${ }^{\mathrm{b}}$ Administered or developed during ICU stay

'Developed during the first week after ICU admission. Severe AKI was defined as Kidney Disease: Improving Global Outcomes (KDIGO) stages 2 and 3

${ }^{\mathrm{d} O d d s}$ ratio represents the increase in risk per $10,000 \mathrm{ng} / \mathrm{mg}$ increase in $\mathrm{uCys} \mathrm{C} / \mathrm{uCr}$

eAfter adjustment for illness severity

${ }^{\mathrm{f}}$ After adjustment for body weight

${ }^{9}$ After adjustment for body weight and illness severity

${ }^{\mathrm{h}}$ After adjustment for body weight, illness severity, MV, and AKI stage 
Table 7 Performance of urinary cystatin C and clinical variables for prediction of ICU mortality $(n=510)$

\begin{tabular}{|c|c|c|c|c|c|c|c|c|}
\hline & AUC & $95 \% \mathrm{Cl}$ & $P$ value & Optimal cutoff value & Sensitivity & Specificity & LR+ & LR- \\
\hline Illness severity ${ }^{a}$, score & 0.74 & $0.68-0.81$ & $<0.001$ & 9.5 & $56.3 \%$ & $79.2 \%$ & 2.7 & 0.55 \\
\hline$M V^{b}$ & 0.73 & $0.65-0.80$ & $<0.001$ & N/A & & & & \\
\hline AKI stage ${ }^{c}$ & 0.60 & $0.50-0.69$ & 0.020 & N/A & & & & \\
\hline Sepsis ${ }^{b}$ & 0.59 & $0.50-0.69$ & 0.033 & N/A & & & & \\
\hline Initial uCysC, ng/mg uCr & 0.76 & $0.69-0.83$ & $<0.001$ & 471.5 & $83.3 \%$ & $60.2 \%$ & 2.1 & 0.28 \\
\hline Peak uCysC, ng/mg uCr & 0.81 & $0.75-0.88$ & $<0.001$ & 1260.0 & $79.2 \%$ & $72.3 \%$ & 2.9 & 0.29 \\
\hline
\end{tabular}

$A K I$ acute kidney injury, $A U C$ the area under the ROC curve, $C l$ confidence interval, $I C U$ intensive care unit, $L R+$ likelihood ratio positive, $L R$ - likelihood ratio negative, $M V$ mechanical ventilation, N/A not applicable, $u C y s C$ urinary cystatin $C, u C r$ urinary creatinine

allness severity was assessed by the score for neonatal acute physiology in critically ill neonates and the pediatric risk of mortality III score in critically ill children

${ }^{\mathrm{b}}$ Administered or developed during ICU stay

'Developed during the first week after ICU admission

higher in patients positive for both $\mathrm{uCysC}(+) / \mathrm{AKI}(+)$ than in patients with $\mathrm{uCysC}(-) / \mathrm{AKI}(-)(P<0.001)$ and in patients with $\mathrm{uCysC}(-) / \mathrm{AKI}(+)$ status $(P=0.011)$. However, the difference between $\mathrm{uCysC}(+) / \mathrm{AKI}(+)$ and $\mathrm{uCysC}(-) / \mathrm{AKI}(+)$ did not remain significant after controlling for body weight and illness severity $(P=0.077)$, as shown in Table 8 . In addition, the first $\mathrm{uCysC}$ level was below the cutoff value of $1260 \mathrm{ng} / \mathrm{mg} \mathrm{uCr}$ in 388 of 510 patients. Among the 388 patients, 48 developed $\mathrm{uCysC}(+)$ status with or without AKI, during serial measurements. These patients had significantly higher mortality rates (18.8\%) compared to those with $\mathrm{uCysC}(-)$ status with or without AKI during the first week after ICU admission $(3.1 \%, P=0.001)$. Demographic and clinical

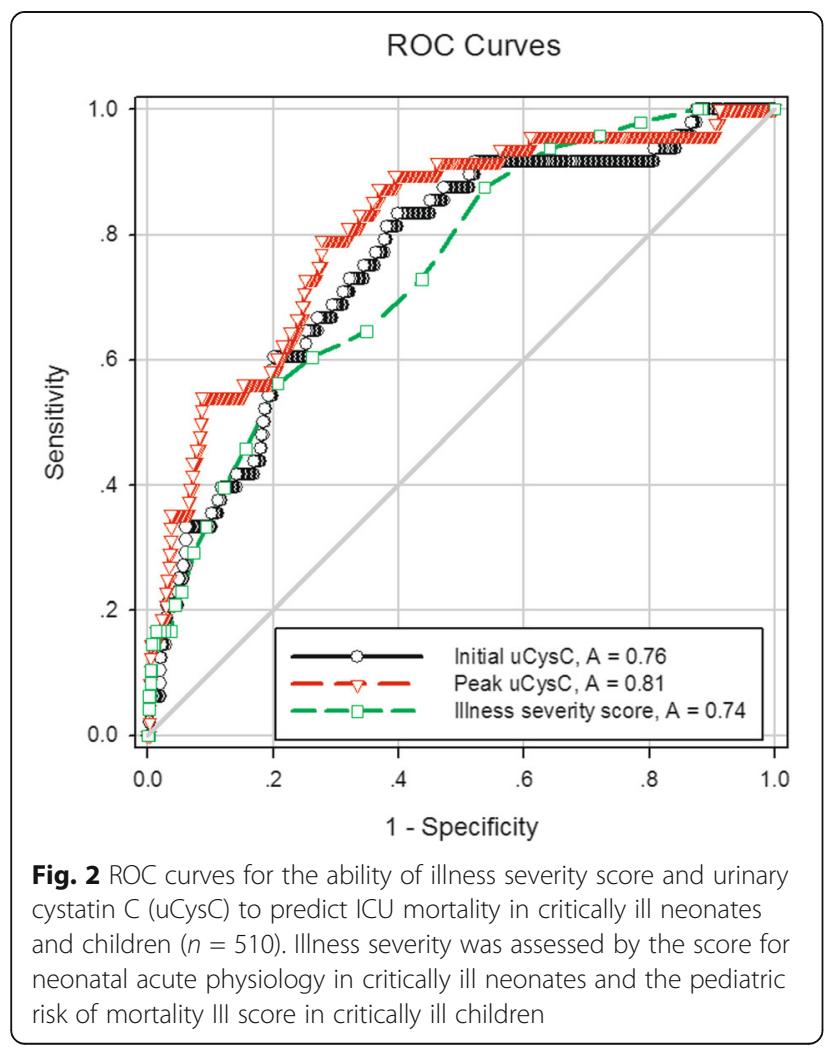

characteristics and outcomes grouped according to $\mathrm{uCysC}$ and AKI status in critically ill neonates and children, respectively, are shown in Additional file 1: Tables S5-S6.

To strengthen the conclusion of association between uCysC-positive subclinical AKI and ICU mortality, the $\mathrm{uCysC}(+) / \mathrm{AKI}(-)$ population was further defined based on different cutoff values of uCysC. Comparisons of mortality rates among the four groups of patients grouped by uCysC/AKI status (defined by the optimal cutoff value of the initial or peak uCysC for predicting AKI, severe AKI, or mortality) in critically ill neonates and children are shown in Additional file 1: Table S7. Demographic and clinical characteristics and outcomes grouped according to uCysC/AKI status, which was defined by the optimal cutoff value of the initial $\mathrm{uCysC}(1788 \mathrm{ng} / \mathrm{mg} \mathrm{uCr})$ for predicting AKI, are displayed in Additional file 1: Table S8.

Furthermore, the analysis of association between uCysC-positive subclinical AKI and mortality is shown in Table 9. The OR for patients with uCysC(+)/AKI(-) status having an increased risk of mortality compared to those with uCysC(-)/AKI(-) status was 12.29 (95\% CI, 4.9230.70; $P<0.001)$. The association remained significant on multivariate logistic regression analysis after adjusting for body weight and illness severity $(\mathrm{AOR}=9.34,95 \% \mathrm{CI}$ 3.55-24.61, $P<0.001)$, as shown in Table 9.

\section{Association between urinary CysC-positive subclinical AKI and secondary outcomes}

There was no significant difference in the duration of MV between patients with uCysC-/AKI- and uCysC + /AKI- $(\mathrm{P}=0.178)$ status. Compared to patients negative for both (uCysC-/AKI-), patients with uCysC(+)/ $\mathrm{AKI}(-)$ spent 2.8 times as long in the ICU $(P<0.001)$. Univariate linear regression analysis further revealed that $\mathrm{uCysC+/AKI}-$ status was significantly associated with a longer ICU stay, compared to uCysC-/AKI- status $(B=$ $0.392, \mathrm{SE}=0.050, P<0.001)$. The association remained significant after adjustment for body weight and illness severity $(B=0.111, \mathrm{SE}=0.045, P=0.015)$ using multivariate linear regression analysis, as shown in Table 9. 
Table 8 Demographic and clinical characteristics and outcomes grouped according to urinary cystatin C and AKI status

\begin{tabular}{|c|c|c|c|c|c|}
\hline & uCysC(-)/ AKI(-) & uCysC(+)/ AKI(-) & uCysC(-)/ AKI(+) & uCysC(+)/ AKI(+) & $P$ value \\
\hline Number & $301(59.0)$ & $130(25.5)$ & $43(8.4)$ & $36(7.1)$ & N/A \\
\hline Body weight, kg & $5.5[3.0-12.0]$ & $2.4[1.5-5.0]^{*}$ & $10.0[3.4-19.0]^{* \#}$ & $5.0[1.4-13.0]^{\#}$ & $<0.001$ \\
\hline Male, $n$ & $184(61.1)$ & $68(52.3)$ & $28(65.1)$ & $24(66.7)$ & 0.216 \\
\hline Illness severity ${ }^{a}$, score & $5[2-7]$ & $8[5-10]^{*}$ & $7[5-11]^{*}$ & $12[8-16.5]^{* \# \&}$ & $<0.001$ \\
\hline$M V, n$ & 77 (25.6) & $42(32.3)$ & $19(44.2)^{*}$ & $24(66.7)^{* \#}$ & $<0.001$ \\
\hline MV duration, days & $0[0-0.38]$ & $0[0-1.10]$ & $0[0-3.96]^{*}$ & $3.16[0-5.65]^{* \# \&}$ & $<0.001$ \\
\hline Severe $A K{ }^{b}, n$ & $0(0)$ & $0(0)$ & $14(32.6)^{* \#}$ & $22(61.1)^{* \# \&}$ & $<0.001$ \\
\hline ICU LOS, hours & $121.0[56.0-228.1]$ & $336.0[132.9-774.0]^{*}$ & $144.0[63.0-288.0]^{\#}$ & $236.0[137.0-917.8]^{* \&}$ & $<0.001$ \\
\hline Death, $n$ & $6(2.0)$ & $26(20.0)^{*}$ & $4(9.3)^{*}$ & $12(33.3)^{*} \&$ & $<0.001^{c}$ \\
\hline
\end{tabular}

Values are median [interquartile range] or number (percentage). $\mathrm{uCys} C(-)$ indicates the absence of tubular injury, and uCys $\mathrm{C}(+)$ indicates the presence of tubular injury defined by the optimal cutoff value of the peak uCysC for predicting mortality $(1260 \mathrm{ng} / \mathrm{mg} \mathrm{uCr})$

$A K I$ acute kidney injury, $I C U$, intensive care unit, LOS length of stay, $M V$ mechanical ventilation, $u C y s C$ urinary cystatin $C$, $u C r$ urinary creatinine

allness severity was assessed by the score for neonatal acute physiology in critically ill neonates and the pediatric risk of mortality III score in critically ill children

${ }^{\mathrm{b}}$ Severe AKI was defined as Kidney Disease: Improving Global Outcomes (KDIGO) stages 2 and 3

${ }^{c} P=0.001$, after adjustment for body weight, illness severity, MV, and severe AKI

${ }^{*} P<0.05$ vs. uCysC(-)/AKI(-). ${ }^{\#} P<0.05$ vs. uCysC(+)/AKI(-). ${ }^{\&} P<0.05$ vs. uCysC(-)/AKI(+)

\section{Discussion}

Our data indicate that subclinical AKI may occur without detectable loss of kidney function and predict worse clinical outcomes, and that urinary CysC is capable of detecting subclinical AKI in critically ill neonates and children.

Urinary CysC was associated with AKI in critically ill neonates and children in this study. The findings further strengthen the evidence for a relationship between elevated uCysC and AKI [11, 19, 20, 30]. Clinical studies investigating the association between $\mathrm{uCysC}$ and mortality have been limited mainly to adult patients $[18,19]$. Studies in general critically ill adults demonstrate that $\mathrm{uCysC}$ is an independent predictor of mortality $[18,19]$. In infants undergoing cardiac surgery with cardiopulmonary bypass, elevated $\mathrm{uCysC}$ in the early postoperative period is associated with a greater risk of death or need for renal replacement therapy [31]. Compared to the previous study in infants undergoing cardiac surgery, our study included a general NICU and PICU population. Nevertheless, our results are in line with the previous study and indicate that

Table 9 Association between urinary cystatin C-positive subclinical AKI and adverse outcomes

\begin{tabular}{ll}
\hline Outcomes & uCysC(+)/AKI(-) vs. uCysC(-)/AKI(-) \\
\hline Mortality, $n$ & OR $12.29(95 \%$ Cl 4.92-30.70), $P<0.001$ \\
& AOR $^{\mathrm{b}} 9.34(95 \%$ Cl 3.55-24.61), $P<0.001$ \\
Length of ICU stay ${ }^{\mathrm{a}}$, hours & $B 0.392($ SE 0.050), $P<0.001$ \\
& $B^{c} 0.111$ (SE 0.045), $P=0.015$ \\
\hline
\end{tabular}

$\mathrm{B}$ is the unstandardized coefficient

$A K I$ acute kidney injury, $A O R$ adjusted $O R, C l$ confidence interval, $I C U$ intensive care unit, $O R$ odds ratio, $S E$ standard error, $u C y s C$ urinary cystatin $C$

${ }^{a} \mathrm{ICU}$ length of stay was log-transformed for linear regression analysis

${ }^{\mathrm{b}}$ After adjustment for body weight and illness severity using multivariate

binary logistic regression analysis

'After adjustment for body weight and illness severity using multivariate linear

regression analysis; body weight was log-transformed due to skewed distribution
$\mathrm{uCys} C$ is a sensitive biomarker for predicting mortality in critically ill neonates and children. For every $1 \%$ increase in uCysC $(10,000 \mathrm{ng} / \mathrm{mg} \mathrm{uCr})$, the odds of mortality increased by $26 \%$. We further demonstrated that the association between uCysC and mortality was independent of $\mathrm{AKI}$ and illness severity. Urinary CysC-positive critically ill neonates and children, with or without clinical AKI diagnosed by the KDIGO criteria, developed worse clinical outcomes.

The major finding in this study was that $25.5 \%$ of patients who had an increased risk of adverse outcomes were identified based on increased $\mathrm{uCysC}$, in the absence of oliguria or increased $\mathrm{sCr}(\mathrm{uCysC}+/ \mathrm{AKI}-)$. It is well-known that increased $\mathrm{sCr}$ is not detected until the glomerular filtration rate is reduced by $>50 \%[15,32]$. AKI can be diagnosed early by damage or functional biomarkers preceding loss of filtration function $[12,13,15$, 33]. Increased uCysC has been demonstrated to reflect renal tubular injury and impairment and predict AKI 24-48 h before sCr [11]. Our data indicate that this substantial group of critically ill patients with positive uCysC who do not fulfill current KIDGO criteria for $\mathrm{AKI}$ are likely to have subclinical AKI, and uCysC can detect subclinical AKI when tubular damage has occurred without oliguria or appreciable increase in $\mathrm{sCr}$.

To our knowledge, this study is the first prospective investigation to determine the association between subclinical AKI and clinical outcomes in critically ill patients. The development of subclinical AKI in critically ill neonates and children is associated with negative clinical outcomes. Our data agree with a previous report conducted in adult patients with cardiorenal syndrome type 1 , which was a retrospective analysis of pooled data from ten prospective observational studies of neutrophil gelatinase-associated lipocalin (NGAL) and demonstrated that in the absence of a diagnostic increases in sCr, NGAL can be 
used to identify patients with subclinical AKI who have an increased risk of adverse outcomes [15]. It is known that $\mathrm{sCr}$ fails to identify likely AKI in some patients who are at an increased risk of death $[9,15]$. The patients with uCysC-positive subclinical AKI were at greater risk of longer ICU stay and mortality compared to those with $\mathrm{uCysC}(-) / \mathrm{AKI}(-)$, suggesting that the concept of subclinical AKI as a diagnosis with promise for improving the management of AKI is clinically relevant and that critically ill neonates and children with subclinical AKI might benefit from early diagnosis and treatment.

In this study, we also found that $8.4 \%$ of the patients had no biomarker evidence of tubular injury but had loss of kidney function ( $\mathrm{uCysC}-/ \mathrm{AKI}+$ ), suggesting that this subgroup of patients who had functional change without damage might have pre-renal azotemia, which is considered a volume-responsive, reversible alteration in kidney function $[8,34]$. These patients had a fourfold increased risk of mortality compared to patients negative for both ( $\mathrm{uCysC}$ -/AKI-). A further study is warranted to explore whether the combined use of $\mathrm{uCysC}$ with $\mathrm{sCr}$ and/or urine output might differentiate between $\mathrm{AKI}$ and pre-renal azotemia.

Moreover, the patients with $\mathrm{uCysC}(+) / \mathrm{AKI}(+)$ had the greatest risk of mortality, which is consistent with findings from the multicenter pooled analysis of the adult studies [15] and supports the concept that subclinical tubular injury precedes detectable decreased kidney function and that when both features occur together, clinical outcomes are worse. Interestingly, our data confirm the potential classification of patients according to the conceptual framework based on a combination of damage and functional biomarkers. The framework is proposed by the Acute Dialysis Quality Initiative (ADQI) conference and provides a potent approach for assessing patients with AKI for diagnosis, staging, differential diagnosis, and prognosis in order to enable clinicians and researchers to most effectively utilize AKI biomarkers [8].

There are a number of limitations to our study. First, the study was not a multicenter study. Nevertheless, the relatively large sample size, with a prospective study design and a protocol with frequent $\mathrm{uCysC}$ measurements, provided adequate power for assessing the association between $\mathrm{uCysC}$ and mortality. Second, the diagnosis of neonatal AKI based on $\mathrm{sCr}$ and urine output is troublesome [27, 35-37]. Serum Cr reflects maternal levels during the first 2-3 days of life and declines progressively with increasing postnatal age to reach a stable neonatal level by 2 weeks of life in neonates with more than 26 weeks of gestational age $[38,39]$. The total body water content and the urine output in neonates are normally greater than those in other populations, suggesting that urine output $\leq 0.5 \mathrm{~mL} / \mathrm{kg} / \mathrm{h}$ is a nonsensitive marker of neonatal AKI [37]. Therefore, the diagnosis and staging of AKI based on neonatal AKI KDIGO may have underestimated the incidence and grade of AKI in neonates $[1,40]$. Moreover, the results from the NICU population seem to be a large driver of the overall study results, which would bias the study towards positive results. Nevertheless, the PICU analyses strengthen the fact that there truly is a signal in the results because the older children do not have this issue to the same extent but still seem to have uCysC-positive subclinical AKI that increases mortality risk. Third, the majority of critically ill children did not have baseline $\mathrm{sCr}$ measurement within 3 months prior to PICU admission. The incidence of AKI may be underestimated when $\mathrm{sCr}$ at PICU admission is used as a baseline; therefore, the lowest $\mathrm{sCr}$ within 2 weeks in the PICU was used as a baseline for patients with elevated $\mathrm{sCr} \geq 1.2 \mathrm{mg} / \mathrm{dL}(106.1 \mu \mathrm{mol} / \mathrm{L})$ at admission. Although it has not been validated in critically ill children, a previous study suggests that AKI incidence is best estimated by choosing the lowest $\mathrm{sCr}$ value within the first week in the ICU as a baseline sCr [29]. Fourth, we did not measure uCysC daily as part of the study, which may underestimate the incidence and grade of AKI and limit our ability to determine the exact time at which the levels of $\mathrm{uCysC}$ rise prior to a $\mathrm{sCr}$ increase. Fifth, there is no specific cutoff value for uCysC to identify AKI and mortality in critically ill neonates and children. We set the $\mathrm{uCysC}$ level of $1260 \mathrm{ng} / \mathrm{mg} \mathrm{uCr}$, which was the optimal cutoff value for $\mathrm{uCysC}$ to predict mortality, as the threshold for identifying tubular injury. The discrepancy in cutoff points between this study and our previous study [20] or between critically ill neonates and children in this study may be related to the fact that we evaluated the predictive accuracy of $\mathrm{uCys} C$ in a general and heterogeneous NICU and PICU population in this study. The level of urinary $\mathrm{CysC}$ was significantly affected by postnatal age [41]. Sixth, the concept of uCysC-positive subclinical AKI has not been validated in different case mixes, especially in patients with higher scores of illness severity. Further multicenter prospective studies are necessary to confirm our findings and define age-related reference values of uCysC for neonates and children.

\section{Conclusions}

Both the initial and the peak levels of $\mathrm{uCysC}$ are significantly associated with AKI and mortality and are independently predictive of ICU mortality in critically ill neonates and children. Subclinical AKI may occur without detectable loss of kidney function. Urinary level of $\mathrm{CysC}$ is a sensitive detector of subclinical AKI, and $\mathrm{uCysC}$-positive subclinical AKI is associated with worse clinical outcomes in critically ill neonates and children. Further randomized and controlled clinical trials are needed to explore whether treatment of subclinical AKI would improve the clinical outcomes in this population. 


\section{Additional files}

Additional file 1: Table S1. Urinary cystatin C levels grouped according to AKI status. Table S2. Association of urinary cystatin C and clinical variables with ICU mortality in critically ill neonates and children, respectively. Table S3. Predictive characteristics of urinary cystatin C at different cutoff values for ICU mortality in critically ill neonates and children. Table S4. Predictive characteristics of urinary cystatin C for ICU mortality in critically ill neonates and children, respectively. Table S5. Demographic and clinical characteristics and outcomes grouped according to urinary cystatin C and AKI status in critically ill neonates. Table S6. Demographic and clinical characteristics and outcomes grouped according to urinary cystatin $\mathrm{C}$ and AKI status in critically ill children. Table S7. Comparison of mortality rates among groups of uCysC/AKI status defined by different cutoff values of urinary cystatin C. Table S8. Demographic and clinical characteristics and outcomes grouped according to urinary cystatin C and AKI status defined by the optimal cutoff value of the initial uCysC for predicting AKI. (DOCX 53 kb)

Additional file 2: Figure S1. Comparison of ICU mortality (A) and the length of ICU stay (B) by quintile of peak value of urinary cystatin C in critically ill neonates and children $(n=510)$. The peak values of urinary Cys $C$ were divided into quintiles, $n=102$ in each group. $P$ value refers to comparison among five groups. ${ }^{*} P<0.05$ vs. the first; ${ }^{\#} P<0.05$ vs. the second; ${ }^{\&} P<0.05$ vs. the third; ${ }^{\$} P<0.05$ vs. the fourth quintile. A Error bars represent the mortality rate and $95 \%$ confidence interval. B Error bars represent the mean and 95\% confidence interval. (TIF $72 \mathrm{~kb}$ )

\section{Abbreviations}

AKI: Acute kidney injury; AOR: Adjusted odds ratio; AUC: Area under the receiver operating characteristic curve; $\mathrm{Cl}$ : Confidence interval; $\mathrm{Cr}$ : Creatinine; CysC: Cystatin C; DIC: Disseminated intravascular coagulation; IQR: Interquartile range; KDIGO: Kidney Disease: Improving Global Outcomes; LOS: Length of stay; LR-: Likelihood ratio negative; LR+: Likelihood ratio positive; MODS: Multiple organ dysfunction syndrome; MV: Mechanical ventilation; NGAL: Neutrophil gelatinase-associated lipocalin; NICU: Neonatal intensive care unit; OR: Odds ratio; PICU: Pediatric intensive care unit; PRISM III: Pediatric risk of mortality III; ROC: Receiver operating characteristic curve; sCr: Serum creatinine; SNAP: Score for neonatal acute physiology; uCysC: Urinary cystatin C

\section{Acknowledgements}

We acknowledge the significant contributions made by Yunqing Sun and Wenjing Li. YS and WL were involved in collection of clinical data and urine samples.

\section{Funding}

This work was supported by grants from the National Natural Science Foundation of China (81741054); JiangSu province's science and technology support program (Social Development BE2016675); Natural science foundation of JiangSu province (BK20171217); Key talent of women's and children's health of JiangSu province (FRC201738); SuZhou clinical key disease diagnosis and treatment technology foundation (LCZX201611). The funders had no role in study design, data collection, preparation of the manuscript, and decision to publish.

\section{Availability of data and materials}

The datasets used and/or analyzed during the current study are available from the corresponding author on reasonable request.

\section{Authors' contributions}

FF carried out data analysis and reviewed the manuscript. XH carried out the experiments. XD and SW collected clinical data and urine samples. ZB and JC supervised data collection and carried out the initial interpretation of data. $J P, X L$, and JW coordinated and supervised data collection and carried out the initial interpretation of data. YL had primary responsibility for study design, data analysis, interpretation of data, and writing of the manuscript. All authors approved the final manuscript as submitted and agree to be accountable for all aspects of the work.

\section{Ethics approval and consent to participate}

This study was approved by the Institutional Review Board at the Children's Hospital of Soochow University, and performed in accordance with the Declaration of Helsinki. Informed parental consent was obtained at enrollment.

\section{Consent for publication}

Not applicable.

\section{Competing interests}

The authors declare that they have no competing interests.

\section{Publisher's Note}

Springer Nature remains neutral with regard to jurisdictional claims in published maps and institutional affiliations.

\section{Author details}

${ }^{1}$ Institute of Pediatric Research, Children's Hospital of Soochow University, Suzhou, JiangSu province, China. 'Department of Nephrology, Children's Hospital of Soochow University, Suzhou, JiangSu province, China. ${ }^{3}$ Pediatric Intensive Care Unit, Children's Hospital of Soochow University, Suzhou, JiangSu province, China.

Received: 14 May 2018 Accepted: 13 September 2018

Published online: 10 October 2018

References

1. Kaddourah A, Basu RK, Bagshaw SM, Goldstein SL, Investigators A. Epidemiology of acute kidney injury in critically ill children and young adults. N Engl J Med. 2017;376:11-20.

2. Alkandari $O$, Eddington KA, Hyder A, Gauvin F, Ducruet T, Gottesman R, Phan V, Zappitelli M. Acute kidney injury is an independent risk factor for pediatric intensive care unit mortality, longer length of stay and prolonged mechanical ventilation in critically ill children: a two-center retrospective cohort study. Crit Care. 2011:15:R146.

3. Sutherland SM, Ji J, Sheikhi FH, Widen E, Tian L, Alexander SR, Ling XB. AKI in hospitalized children: epidemiology and clinical associations in a national cohort. Clin J Am Soc Nephrol. 2013;8:1661-9.

4. Kellum JA, Prowle JR. Paradigms of acute kidney injury in the intensive care setting. Nat Rev Nephrol. 2018;14:217-30.

5. Parikh CR, Mansour SG. Perspective on clinical application of biomarkers in AKI. J Am Soc Nephrol. 2017;28:1677-85.

6. Malhotra R, Siew ED. Biomarkers for the early detection and prognosis of acute kidney injury. Clin J Am Soc Nephrol. 2017;12:149-73.

7. Ostermann M, Philips BJ, Forni LG. Clinical review: biomarkers of acute kidney injury: where are we now? Crit Care. 2012;16:233.

8. Murray PT, Mehta RL, Shaw A, Ronco C, Endre Z, Kellum JA, Chawla LS, Cruz $D$, Ince C, Okusa MD, et al. Potential use of biomarkers in acute kidney injury: report and summary of recommendations from the 10th Acute Dialysis Quality Initiative consensus conference. Kidney Int. 2014:85:513-21.

9. Haase M, Bellomo R, Devarajan P, Schlattmann P, Haase-Fielitz A, Group NMal. Accuracy of neutrophil gelatinase-associated lipocalin (NGAL) in diagnosis and prognosis in acute kidney injury: a systematic review and meta-analysis. Am J Kidney Dis. 2009;54:1012-24

10. Ralib AM, Pickering JW, Shaw GM, Than MP, George PM, Endre ZH. The clinical utility window for acute kidney injury biomarkers in the critically ill. Crit Care. 2014:18:601.

11. Koyner JL, Bennett MR, Worcester EM, Ma Q, Raman J, Jeevanandam V, Kasza KE, O'Connor MF, Konczal DJ, Trevino S, et al. Urinary cystatin C as an early biomarker of acute kidney injury following adult cardiothoracic surgery. Kidney Int. 2008;74:1059-69.

12. Haase M, Kellum JA, Ronco C. Subclinical AKI-an emerging syndrome with important consequences. Nat Rev Nephrol. 2012;8:735-9.

13. Ronco C, Kellum JA, Haase M. Subclinical AKI is still AKI. Crit Care. 2012;16:313.

14. Moledina DG, Parikh CR. Phenotyping of acute kidney injury: beyond serum creatinine. Semin Nephrol. 2018:38:3-11.

15. Haase M, Devarajan P, Haase-Fielitz A, Bellomo R, Cruz DN, Wagener G, Krawczeski CD, Koyner JL, Murray P, Zappitelli M, et al. The outcome of neutrophil gelatinase-associated lipocalin-positive subclinical acute kidney injury: a multicenter pooled analysis of prospective studies. J Am Coll Cardiol. 2011;57:1752-61. 
16. Conti M, Moutereau S, Zater M, Lallali K, Durrbach A, Manivet P, Eschwege $\mathrm{P}$, Loric S. Urinary cystatin $\mathrm{C}$ as a specific marker of tubular dysfunction. Clin Chem Lab Med. 2006;44:288-91.

17. Herget-Rosenthal S, van Wijk JA, Brocker-Preuss M, Bokenkamp A. Increased urinary cystatin $C$ reflects structural and functional renal tubular impairment independent of glomerular filtration rate. Clin Biochem. 2007;40:946-51.

18. Endre ZH, Pickering JW, Walker RJ, Devarajan P, Edelstein CL, Bonventre JV, Frampton CM, Bennett MR, Ma Q, Sabbisetti VS, et al. Improved performance of urinary biomarkers of acute kidney injury in the critically ill by stratification for injury duration and baseline renal function. Kidney Int. 2011;79:1119-30.

19. Nejat M, Pickering JW, Walker RJ, Westhuyzen J, Shaw GM, Frampton CM, Endre $\mathrm{ZH}$. Urinary cystatin $\mathrm{C}$ is diagnostic of acute kidney injury and sepsis, and predicts mortality in the intensive care unit. Crit Care. 2010;14:R85.

20. Li Y, Fu C, Zhou X, Xiao Z, Zhu X, Jin M, Li X, Feng X. Urine interleukin-18 and cystatin- $C$ as biomarkers of acute kidney injury in critically ill neonates. Pediatr Nephrol. 2012;27:851-60.

21. Li Y, Li X, Zhou X, Yan J, Zhu X, Pan J, Jin M, Zhu X, Feng X, Xiao Z. Impact of sepsis on the urinary level of interleukin-18 and cystatin C in critically ill neonates. Pediatr Nephrol. 2013;28:135-44.

22. Li Y, Wang J, Bai Z, Chen J, Wang X, Pan J, Li X, Feng X. Early fluid overload is associated with acute kidney injury and PICU mortality in critically ill children. Eur J Pediatr. 2016;175:39-48.

23. Chen J, Li X, Bai Z, Fang F, Hua J, Li Y, Pan J, Wang J, Feng X, Li Y. Association of fluid accumulation with clinical outcomes in critically ill children with severe sepsis. PLoS One. 2016;11:e0160093.

24. Richardson DK, Gray JE, McCormick MC, Workman K, Goldmann DA. Score for Neonatal Acute Physiology: a physiologic severity index for neonatal intensive care. Pediatrics. 1993;91:617-23.

25. Pollack MM, Patel KM, Ruttimann UE. PRISM III: an updated Pediatric Risk of Mortality score. Crit Care Med. 1996;24:743-52.

26. Li Y, Yan J, Li M, Xiao Z, Zhu X, Pan J, Li X, Feng X. Addition of SNAP to perinatal risk factors improves the prediction of bronchopulmonary dysplasia or death in critically ill preterm infants. BMC Pediatr. 2013;13:138.

27. Selewski DT, Charlton JR, Jetton JG, Guillet R, Mhanna MJ, Askenazi DJ, Kent AL. Neonatal acute kidney injury. Pediatrics. 2015;136:e463-73.

28. Mehta RL, Kellum JA, Shah SV, Molitoris BA, Ronco C, Warnock DG, Levin A. Acute Kidney Injury N. Acute Kidney Injury Network: report of an initiative to improve outcomes in acute kidney injury. Crit Care. 2007;11:R31.

29. Pickering JW, Endre ZH. Back-calculating baseline creatinine with MDRD misclassifies acute kidney injury in the intensive care unit. Clin J Am Soc Nephrol. 2010;5:1165-73.

30. Askenazi DJ, Koralkar R, Hundley HE, Montesanti A, Parwar P, Sonjara S, Ambalavanan N. Urine biomarkers predict acute kidney injury in newborns. J Pediatr. 2012;161:270-5 e1.

31. Hazle MA, Gajarski RJ, Aiyagari R, Yu S, Abraham A, Donohue J, Blatt NB. Urinary biomarkers and renal near-infrared spectroscopy predict intensive care unit outcomes after cardiac surgery in infants younger than 6 months of age. J Thorac Cardiovasc Surg. 2013;146:861-7 e1.

32. Herget-Rosenthal S, Marggraf G, Husing J, Goring F, Pietruck F, Janssen O, Philipp T, Kribben A. Early detection of acute renal failure by serum cystatin C. Kidney Int. 2004;66:1115-22.

33. Nehus E, Kaddourah A, Bennett M, Pyles O, Devarajan P. Subclinical kidney injury in children receiving nonsteroidal anti-inflammatory drugs after cardiac surgery. J Pediatr. 2017;189:175-80.

34. Schrier RW. Diagnostic value of urinary sodium, chloride, urea, and flow. J Am Soc Nephrol. 2011;22:1610-3.

35. Jetton JG, Askenazi DJ. Update on acute kidney injury in the neonate. Curr Opin Pediatr. 2012:24:191-6.

36. Carmody JB, Swanson JR, Rhone ET, Charlton JR. Recognition and reporting of AKI in very low birth weight infants. Clin J Am Soc Nephrol. 2014;9:2036-43.

37. Liborio AB, Branco KM. Torres de Melo Bezerra C. Acute kidney injury in neonates: from urine output to new biomarkers. Biomed Res Int. 2014;2014: 601568.

38. Drukker A, Guignard JP. Renal aspects of the term and preterm infant: a selective update. Curr Opin Pediatr. 2002;14:175-82.

39. Gallini F, Maggio L, Romagnoli C, Marrocco G, Tortorolo G. Progression of renal function in preterm neonates with gestational age $<$ or $=32$ weeks. Pediatr Nephrol. 2000;15:119-24.
40. Jin K, Murugan R, Sileanu FE, Foldes E, Priyanka P, Clermont G, Kellum JA. Intensive monitoring of urine output is associated with increased detection of acute kidney injury and improved outcomes. Chest. 2017;152:972-9.

41. Saeidi B, Koralkar R, Griffin RL, Halloran B, Ambalavanan N, Askenazi DJ. Impact of gestational age, sex, and postnatal age on urine biomarkers in premature neonates. Pediatr Nephrol. 2015;30:2037-44.
Ready to submit your research? Choose BMC and benefit from:

- fast, convenient online submission

- thorough peer review by experienced researchers in your field

- rapid publication on acceptance

- support for research data, including large and complex data types

- gold Open Access which fosters wider collaboration and increased citations

- maximum visibility for your research: over $100 \mathrm{M}$ website views per year

At $\mathrm{BMC}$, research is always in progress.

Learn more biomedcentral.com/submissions 